\title{
Fractional Measure-dependent Nonlinear Second-order Stochastic Evolution Equations with Poisson Jumps
}

https://doi.org/10.1515/msds-2018-0005

Received August 21, 2017; accepted April 7, 2018

\begin{abstract}
This paper focuses on a nonlinear second-order stochastic evolution equations driven by a fractional Brownian motion ( $\mathrm{fBm}$ ) with Poisson jumps and which is dependent upon a family of probability measures. The global existence of mild solutions is established under various growth conditions, and a related stability result is discussed. An example is presented to illustrate the applicability of the theory.
\end{abstract}

Keywords: Stochastic evolution equation; fractional Brownian motion; second-order equation; Poisson jumps; cosine family

MSC: 60H05; 60H15; 60H20; 35R09

\section{Introduction}

Second-order stochastic evolution equations have been the subject of numerous papers over the past several decades (See $[20,23,24,26,27,29])$. In this paper, we extend existing work in this area by considering a stochastic second-order evolution equations driven by a fractional Brownian motion ( $\mathrm{fBm}$ ) in which the forcing terms are dependent on the probability law of the state function and in which Poisson jumps are present. Specifically, let $\mathscr{D}$ be a bounded domain in $\mathbb{R}^{N}$ with smooth boundary $\partial \mathscr{D}$. Consider the following initial boundary value problem:

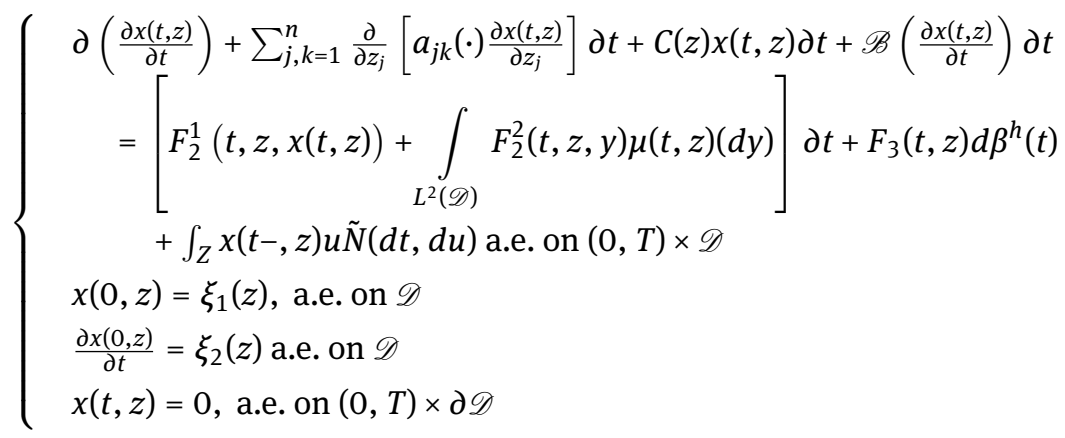

where $z=\left\langle z_{1}, \ldots, z_{n}\right\rangle \in \mathscr{D} ; x:[0, T] \times \mathscr{D} \rightarrow \mathbb{R}, F_{2}^{1}:[0, T] \times \mathscr{D} \times \mathbb{R} \rightarrow \mathbb{R} ; F_{2}^{2}:[0, T] \times \mathscr{D} \times L^{2}(\mathscr{D}) \rightarrow L^{2}(\mathscr{D})$; $F_{3}:[0, T] \times \mathscr{D} \rightarrow \mathfrak{L}\left(\mathbb{R}, L^{2}(\mathscr{D})\right) ; a_{j k}: \mathscr{D} \rightarrow \mathbb{R}(1 \leq j, k \leq n) ; C: \mathscr{D} \rightarrow \mathbb{R} ; \mathscr{B}: L^{2}(\mathscr{D}) \rightarrow L^{2}(\mathscr{D}) ;$ and $\mu(t, \cdot) \in$ $\wp \lambda^{2}\left(L^{2}(\mathscr{D})\right)$ is the probability law of $x(t, \cdot)$. In addition, $\left\{\beta^{h}(t): 0 \leq t \leq T\right\}$ is a real fBm and $\{p(t): t \in[0, T]\}$ is a Poisson point process, independent of $\beta^{h}(t)$, taking values in $[0, \infty)$ with a $\sigma$-finite characteristic measure

\footnotetext{
^Corresponding Author: Mark A. McKibben: Department of Mathematics, West Chester University, 25 University Avenue, West Chester, PA, 19383, E-mail: mmckibben@wcupa.edu

Micah Webster: Center for Data, Mathematical, and Computational Sciences, Goucher College, 1021 Dulaney Valley Road, Baltimore, MD, 21286, E-mail: micah.webster@goucher.edu
} 
$\lambda(d u)$ and $Z \in \mathfrak{B}([0, \infty) \backslash\{0\})$. The Poisson random measure induced by $p(\cdot)$ is denoted by $N(d t, d u)$, and the compensated Poisson measure by

$$
\tilde{N}(d t, d u)=N(d t, d u)-\lambda(d u) d t .
$$

We shall study the global existence and stability properties of mild solutions of this IBVP by considering a more abstract stochastic evolution equation of the form

$$
\left\{\begin{aligned}
d\left[x^{\prime}(t)-f_{1}(t, x(t))\right]= & {\left[B x^{\prime}(t)+A x(t)+f_{2}(t, x(t), \mu(t))\right] d t+f_{3}(t) d \beta^{h}(t) } \\
& +\int_{Z} f_{4}(t, x(t-), \eta) \tilde{N}(d t, d \eta), \quad 0 \leq t \leq T \\
& x(0)=x_{0}, \quad x^{\prime}(0)=x_{1}, \\
& \mu(t)=\text { probability distribution of } x(t)
\end{aligned}\right.
$$

in a separable Hilbert Space $H$. (By the probability distribution of $x(t)$, we mean $\mu(t)(\mathcal{A})=P(\{\omega \in \Omega$ : $x(t, \omega) \in \mathcal{A}\}$ ) for each $\mathcal{A} \in \mathfrak{B}(H)$, where $\mathfrak{B}(H)$ stands for the Borel class on $H$.) Here $A: D(A) \subset H \rightarrow H$ is a linear (possibly unbounded) operator which generates a strongly continuous cosine family $\{C(t): t \geq 0\}$; $B: H \rightarrow H$ is a bounded linear operator; $f_{1}:[0, T] \times H \rightarrow H ; f_{2}:[0, T] \times H \times \wp_{\lambda^{2}}(H) \rightarrow H ; f_{3}:[0, T] \rightarrow \mathfrak{L}(K, H)$ is a bounded, strongly measurable mapping (where $K$ is a real separable Hilbert Space and $\mathfrak{L}(K, H)$ denotes the space of Hilbert-Schmidt operators from $K$ into $H$ with norm $\left.\|\cdot\|_{\mathfrak{L}(K, H)}\right) ; f_{4}:[0, T] \times H \times Z \rightarrow H ;\left\{\beta^{h}(t)\right.$ : $t \geq 0\}$ is a $K$-valued fBm with Hurst parameter $h \in\left(\frac{1}{2}, 1\right) ; \tilde{N}(d t, d \eta)$ is a compensated measure induced by a Poisson point process, which is independent of the $\mathrm{fBm}$; and $x_{0}, x_{1}$ are $\mathfrak{F}_{0}$-measurable random variables independent of $\beta^{h}$. (See Section 2 for notation and spaces.)

One improvement we have incorporated into the study concerns how noise is incorporated into the evolution equation. The stochastic processes $\{x(\alpha t): 0 \leq t \leq T\}$ arising in some applications exhibits a selfsimilarity property in the sense that the process $\{x(\alpha t): 0 \leq t \leq T\}$ and $\left\{\alpha^{h} x(t): 0 \leq t \leq T\right\}$ have the same probability distribution (see [21]). Concrete data extracted from various applications suggest that values of $h$ other than $\frac{1}{2}$ are better to use when modeling such phenomena. Doing so introduces a technical complication since the usual Itô stochastic calculus cannot be used because the noise process is not a semimartingale when $h \neq \frac{1}{2}$. This leads to using a so-called fractional Brownian motion in place of a standard Wiener process to model the noise; the details of the stochastic calculus related to fBm can be found in $[3,10,14,22]$.

A second improvement concerns the dependence on the probability law. Often, a more accurate model of such phenomena can be formulated by allowing the nonlinear perturbations to depend on the probability law of the state process at time $t$. A prototypical example in the finite-dimensional setting would be an interacting $M$-particle system in which (1.2) describes the dynamics of the particles $x_{1}, \ldots, x_{M}$ moving in the space $H$ in which the probability measure $\mu(t)$ is taken to be the empirical measure $\mu_{M}(t)=(1 / M) \sum_{k=1}^{M} \delta_{x_{k}(t)}$, where $\delta_{x_{k}(t)}$ denotes the Dirac measure. Researchers have investigated related models concerning diffusion processes in the finite-dimensional case $[8,9,25]$. The infinite dimensional version of such models in a Hilbert space setting has only recently been examined $[1,15]$.

A third improvement relates to incorporating Poisson jumps into the IBVP. Including such jumps in stochastic evolution equations is of particular interest when modeling phenomena for which the underlying randomness of the system contains jumps. Such examples arise in finance, economics, physics, ecology, and biology to name a few. Researcher have studied such models (see [2, 19, 27, 29]), but to the authors' knowledge models involving Poisson jumps, fBm, and dependence on the probability law of the state process have not been investigated.

The following is the outline of the paper. First, we make precise the necessary notation and function spaces, and gather certain preliminary results in Section 2. The main existence and uniqueness results are discussed in Section 3 and a stability result is provided in Section 4. Finally, we return to the motivating IBVP in Section 5 to illustrate the applicability of the general theory. 


\section{Preliminaries}

For details on $\mathrm{fBm}$, stochastic analysis, and abstract differential equations, we refer the reader to $[3,4,6$, $16,28]$ and the references therein. Throughout this paper, let $\left(H,\|\cdot\|,\langle\cdot, \cdot\rangle_{H}\right)$ and $\left(K,\|\cdot\|_{K},\langle\cdot, \cdot\rangle_{K}\right)$ be real separable Hilbert spaces, where the complete orthonormal basis for $U$ is denoted by $\left\{e_{j} \mid j=1,2, \ldots\right\}$. Furthermore, we assume that $\left(\Omega, \mathfrak{F},\left\{\mathfrak{F}_{t}\right\}_{t \geq 0}, P\right)$ is a complete filtered probability space for which the filtration is a right continuous increasing family and $\mathfrak{F}_{0}$ contains all $P$-null sets. Suppose that $\{p(t): t \geq 0\}$ is a $\sigma$-finite stationary $\mathfrak{F}_{t}$-adapted Poisson point process taking values in a measurable space $(U, \mathfrak{B}(U))$. We define the Poisson random measure induced by $p(\cdot)$ as $N\left(\left(s_{1}, s_{2}\right], Z\right)=\sum_{s \in\left(s_{1}, s_{2}\right]} \mathbf{1}_{Z}(p(s))$ for any $\left.Z \in \mathfrak{B}(U \backslash\{0\})\right)$, and thus, we may define the compensated Poisson random measure, denoted by $\tilde{N}$, as

$$
\tilde{N}(d t, d \eta)=N(d t, d \eta)-v(d \eta)
$$

where $v$ is the characteristic measure of $N$.

There are different ways to construct a fBm process and a $H$-valued stochastic integral driven by $\mathrm{fBm}$. We use the approach from $[3,17]$. Let $\left\{\beta_{j}^{h}(t) \mid t \geq 0\right\}_{j=1}^{\infty}$ be a sequence of independent, one-dimensional fBms with Hurst parameter $h \in(1 / 2,1)$ such that for all $j=1,2, \ldots$ the following hold:

1. $\beta_{j}^{h}(0)=0$,

2. $E\left[\beta_{j}^{h}(t)-\beta_{j}^{h}(s)\right]^{2}=|t-s|^{2 h} v_{j}$,

3. $E\left[\beta_{j}^{h}(1)\right]^{2}=v_{j}>0$,

4. $\sum_{j=1}^{\infty} v_{j}<\infty$,

where $E(Y)=\int_{\Omega} Y d P$ is the expected value of a random variable $Y$. In such case,

$$
\sum_{j=1}^{\infty} E\left\|\beta_{j}^{h}(t) e_{j}\right\|_{K}^{2}=t^{2 h} \sum_{j=1}^{\infty} v_{j}<\infty
$$

So, the following definition is meaningful.

Definition 2.1. For every $t \geq 0, \beta^{h}(t)=\sum_{j=1}^{\infty} \beta_{j}^{h}(t) e_{j}$ is a $K$-valued $\mathrm{fBm}$, where the convergence is taken in the mean-square sense.

The covariance operator of $\left\{\beta^{h}(t): t \geq 0\right\}$ is a positive nuclear operator $Q$ such that

$$
\operatorname{tr} Q(t, s)=\frac{1}{2} \sum_{j=1}^{\infty} v_{j}\left[t^{2 h}+s^{2 h}-|t-s|^{2 h}\right] .
$$

(See $[3,14]$.) We assume that the filtration is generated by the $\mathrm{fBm}$ process $\beta^{h}(\cdot)$, the Poisson point process $p(\cdot)$ and is augmented, that is,

$$
\mathfrak{F}_{t}=\sigma\left\{\beta^{h}(s): s \leq t\right\} \vee \sigma\{N((0, s], Z): s \leq t, Z \in \mathfrak{B}(U \backslash\{0\})\} \vee \mathscr{N}, t \geq 0,
$$

where $\mathscr{N}$ is the class of $P$-null sets.

Next, we outline the discussion leading to the definition of the stochastic integral associated with $\left\{\beta^{h}(t)\right.$ : $t \geq 0\}$ for bounded, strongly measurable functions. Assume that $f_{3}:[0, T] \rightarrow \mathfrak{L}(K, H)$ is a simple function; that is, there exists $\left\{g_{i}: i=1, \ldots, n\right\} \subset \mathbb{R}$ such that

$$
f_{3}(t)=g_{i}, \quad \forall t_{i-1} \leq t \leq t_{i},
$$

where $0=t_{0}<t_{1}<\cdots<t_{n-1}<t_{n}=T$ and $\max _{1 \leq i \leq n}\left\|g_{i}\right\|_{\mathfrak{L}(K, H)}=L$. 
Definition 2.2. The $H$-valued stochastic integral $\int_{0}^{T} f_{3}(t) d \beta^{h}(t)$ is defined by

$$
\begin{aligned}
\int_{0}^{T} f_{3}(t) d \beta^{h}(t) & =\sum_{j=1}^{\infty}\left(\int_{0}^{T} f_{3}(t) e_{j} d \beta_{j}^{h}(t)\right) \\
& =\sum_{j=1}^{\infty}\left(\sum_{i=1}^{\infty} g_{i} e_{j}\left[\beta_{j}^{h}\left(t_{i}\right)-\beta_{j}^{h}\left(t_{i-1}\right)\right]\right) .
\end{aligned}
$$

This integral is well-defined because

$$
E\left\|\int_{0}^{T} f_{3}(t) d \beta^{h}(t)\right\|_{H}^{2} \leq L^{2} T^{2 h} \sum_{j=1}^{\infty} v_{j}<\infty .
$$

A standard density argument can be used to extend Definition 2.2 to the case of a general bounded, strongly measurable integrand because the set of simple functions is dense in the space of bounded, strongly measurable $\mathfrak{L}(K, H)$-valued functions. The following spaces of measures coincide with those used in [1]; we recall them here for convenience. First, $\mathfrak{B}(H)$ stands for the Borel class on $H$ and $\wp(H)$ represents the space of all probability measures defined on $\mathfrak{B}(H)$ equipped with the weak convergence topology. Let $\lambda(x)=1+\|x\|$. Define the space

$$
\begin{aligned}
\mathscr{C}=\{\varphi: H & \rightarrow H: \varphi \text { is continuous and } \\
\|\varphi\|_{\mathscr{C}} & \left.=\sup _{x \in H} \frac{\|\varphi(x)\|}{\lambda^{2}(x)}+\sup _{x \neq y \in H} \frac{\|\varphi(x)-\varphi(y)\|}{\|x-y\|}<\infty\right\},
\end{aligned}
$$

and for $p \geq 1$, let

$$
\begin{gathered}
\wp_{\gamma^{p}}^{s}(H)=\{m: H \rightarrow \mathbb{R}: m \text { is a signed measure on } H \text { such that } \\
\left.\|m\|_{\lambda^{p}}=\int_{H} \lambda^{p}(x)|m|(d x)<\infty\right\},
\end{gathered}
$$

where $|m|=m^{+}+m^{-}, m=m^{+}-m^{-}$is the is the Jordan decomposition of $m$. Then, we can define the space $\wp_{\lambda^{2}}(H)=\wp_{\lambda^{2}}^{S} \cap \wp(H)$ equipped with the metric $\rho$ given by

$$
\rho\left(v_{1}, v_{2}\right)=\sup \left\{\int_{H} \varphi(x)\left(v_{1}-v_{2}\right)(d x):\|\varphi\|_{\mathscr{C}} \leq 1\right\} .
$$

It is known that $\left(\wp_{\lambda^{2}}(H), \rho\right)$ is a complete metric space. The space of all càdlàg $\wp_{\lambda^{2}}(H)$-valued functions defined on $[0, T]$, denoted $\mathscr{C}_{\lambda^{2}}=D\left([0, T] ;\left(\wp_{\lambda^{2}}(H), \rho\right)\right)$, is a metric space when equipped with

$$
D_{T}\left(v_{1}, v_{2}\right)=\sup _{t \in[0, T]} \rho\left(v_{1}(t), v_{2}(t)\right), \quad \forall v_{1}, v_{2} \in \mathscr{C}_{\lambda^{2}},
$$

and is a complete metric space when endowed with the complete Skorohod metric, $S_{T}$, given by

$$
S_{T}\left(v_{1}, v_{2}\right)=\inf _{\lambda \in \Lambda}\left[\sup _{0 \leq s<t<T}\left|\log \left(\frac{\lambda(t)-\lambda(s)}{t-s}\right)\right|+\sup _{t \in[0, T]} \rho\left(v_{1}(t), v_{2}(\lambda(t))\right)\right],
$$

for all $v_{1}, v_{2} \in \mathscr{C}_{\lambda^{2}}$ and where $\Lambda$ is the usual set of all strictly increasing continuous mappings of [0,T] onto itself. Furthermore, we define the space

$$
X_{T, 2}=\left\{z \in D\left([0, T] ; L^{2}(\Omega ; H)\right): z \text { is } \mathfrak{F} t_{\text {-adapted and } \left.\|z\|_{X_{T, 2}}<\infty\right\}},\right.
$$

which is a subspace of all càdlàg $L^{2}(\Omega ; H)$-valued mappings and is a Banach space itself when equipped with the norm

$$
\|z\|_{X_{T, 2}}=\sup _{0 \leq t \leq T}\left(E\|z\|^{2}\right)^{\frac{1}{2}}
$$

Next, we recall some facts about cosine families of operators. For more details, see [12, 26, 30-32]. 
Definition 2.3. The one-parameter family $\{C(t): t \in \mathbb{R}\} \subset \mathfrak{B} \mathfrak{L}(H)$ satisfying

1. $C(0)=I$,

2. $C(t) x$ is continuous in $t$ on $\mathbb{R}$, for all $x \in H$,

3. $C(t+s)+C(t-s)=2 C(t) C(s)$, for all $t, s \in \mathbb{R}$,

is a strongly continuous cosine family. The corresponding strongly continuous sine family $\{S(t): t \in \mathbb{R}\} \subset$ $\mathfrak{B} \mathfrak{L}(H)$ is defined by $S(t) x=\int_{0}^{t} C(s) x d s$, for all $t \in \mathbb{R}$ and for all $x \in H$.

Definition 2.4. The (infinitesimal) generator $A: H \rightarrow H$ of $\{C(t): t \in \mathbb{R}\}$ is given by

$$
A x=\left.\frac{d^{2}}{d t^{2}} C(t) x\right|_{t=0},
$$

for all $x \in D(A)=\left\{x \in H: C(\cdot) x \in C^{2}(\mathbb{R} ; H)\right\}$.

It is known that the infinitesimal generator $A$ is a closed, densely-defined operator on $H$ (see [11]). Cosine and corresponding sine families and their generators satisfy the following properties:

Proposition 2.5. Suppose that $A$ is the infinitesimal generator of a cosine family of operators $\{C(t): t \in \mathbb{R}\}$ (cf. Definition 2.3). Then, the following hold:

1. There exist $M_{A} \geq 1$ and $\alpha \geq 0$ such that $\|C(t)\|_{\mathfrak{B} \mathfrak{L}(H)} \leq M_{A} e^{\alpha|t|}$ and hence, $\|S(t)\|_{\mathfrak{B} \mathfrak{L}(H)} \leq M_{A} e^{\alpha|t|}$, for all $t>0$

2. $A \int_{s}^{r} S(u) x d u=[C(r)-C(s)] x$, for all $0 \leq s \leq r<\infty$ and $x \in H$;

3. There exists $N \geq 1$ such that $\|S(t)-S(r)\|_{\mathfrak{B} \mathfrak{L}(H)} \leq N\left|\int_{r}^{t} e^{\alpha|s|} d s\right|$, for all $0 \leq r \leq t<\infty$.

The Uniform Boundedness Principle, together with Proposition 2.5, implies that both $\{C(t): t \in[0, T]\}$ and $\{S(t): t \in[0, T]\}$ are uniformly bounded by $\bar{M}=M_{A} e^{\alpha|T|}$. This, in turn, implies that there exists $N_{S}>0$ such that

$$
\|S(t)-S(r)\|_{\mathfrak{B} \mathfrak{L}(H)} \leq N_{S}|t-r|
$$

for all $0 \leq r \leq t \leq T$ by Proposition 2.5 (3).

In addition to the familiar Young, Hölder, and Minkowski inequalities, the inequality of the form $\left(\sum_{i=1}^{n} a_{i}\right)^{m} \leq m^{n-1} \sum_{i=1}^{n} a_{i}^{m}$, where $a_{i}$ is a nonnegative constant $(\mathrm{i}=1,2, \ldots, \mathrm{n})$ and $m, n \in \mathbb{N}$, will be used to establish various estimates. Finally, the following Bihari and, so-called, extended Bihari inequalities (see [29]) play important roles in the proofs of certain results.

Lemma 2.6. Let $T>0, u_{0} \geq 0$, and $u(t), v(t)$ be continuous functions on $[0, T]$. Let $\theta:[0, \infty) \rightarrow[0, \infty)$ be a concave continuous and nondecreasing function such that theta $(r)>0$ for $r>0$. If

$$
u(t) \leq u_{0}+\int_{0}^{t} v(s) \theta(u(s)) d s,
$$

for all $0 \leq t \leq T$, then

$$
u(t) \leq G^{-1}\left(G\left(u_{0}\right)+\int_{0}^{t} v(s) d s\right),
$$

for all $t \in[0, T]$ and $G\left(u_{0}\right)+\int_{0}^{t} v(s) d s \in \operatorname{Dom}\left(G^{-1}\right)$, where $G(r)=\int_{1}^{r} \frac{d s}{\theta(s)}, r \geq 0$, and $G^{-1}$ is the inverse of $G$. Furthermore, if $u_{0}=0$ and $\int_{0}^{\infty} \frac{d s}{\theta s}=\infty$, then $u(t)=0$ for all $0 \leq t \leq T$. 
Lemma 2.7. Let the assumptions of Lemma 2.6 hold. If

$$
u(t) \leq u_{0}+\int_{t}^{T} v(s) \theta(u(s)) d s
$$

for all $0 \leq t \leq T$, then

$$
u(t) \leq G^{-1}\left(G\left(u_{0}\right)+\int_{t}^{T} v(s) d s\right),
$$

for all $t \in[0, T]$ and $G\left(u_{0}\right)+\int_{t}^{T} v(s) d s \in \operatorname{Dom}\left(G^{-1}\right)$, where $G(r)=\int_{1}^{r} \frac{d s}{\theta(s)}, r \geq 0$, and $G^{-1}$ is the inverse of $G$.

Corollary 2.8. Let the assumptions of Lemma 2.6 hold and $v(t) \geq 0$ for $t \in[0, T]$. If for all $\epsilon>0$, there exists $t_{1} \geq 0$, for all $0 \leq u_{0}<\epsilon, \int_{t_{1}}^{T} v(s) d s \leq \int_{u_{0}}^{\epsilon} \frac{d s}{\theta(s)}$ holds. Then for every $t \in\left[t_{1}, T\right], u(t) \leq \epsilon$.

\section{Existence and Uniqueness Results}

We consider mild solutions of (1.2) in the following sense:

Definition 3.1. For a given $\mu \in \mathscr{C}_{\lambda^{2}}$, a cádlág stochastic process $x_{\mu}:[0, T] \rightarrow H$ is a parametrized mild solution of (1.2) if

1. $x_{\mu}(t)$ is $\mathfrak{F}_{t}$-adapted, for each $0 \leq t \leq T$,

2. $\int_{0}^{T}\left\|x_{\mu}(s)\right\|^{2} d s<\infty$, almost surely $[P]$,

3. $x_{\mu}(t)$ satisfies the formula

$$
\begin{aligned}
x(t)= & S(t)\left(x_{1}-f_{1}\left(0, x_{0}\right)\right)+(C(t)-S(t) B) x_{0} \\
& +\int_{0}^{t} C(t-s)\left[B x(s)+f_{1}(s, x(s))\right] d s+\int_{0}^{t} S(t-s) f_{2}(s, x(s), \mu(s)) d s \\
& +\int_{0}^{t} S(t-s) f_{3}(s) d \beta^{\mathcal{H}}(s)+\int_{0}^{t} S(t-s) \int_{Z} f_{4}(s, x(s-), \eta) \tilde{N}(d s, d \eta) .
\end{aligned}
$$

In addition, if $\mu$ is the probability law of $x_{\mu}$, then $x_{\mu}$ is the mild solution of (1.2).

For our first result, we impose the following conditions on (1.2):

(H1) $A$ is the infinitesimal generator of a strongly continuous cosine family $\{C(t): t \geq 0\}$ on $H$. The cosine family and corresponding strongly continuous sine family $\{S(t): t \geq 0\}$ on $H$ satisfy

$$
\sup _{0 \leq t \leq T}\left[\|S(t)\|_{\mathfrak{B} \mathfrak{L}(H)}^{2}+\|C(t)\|_{\mathfrak{B} \mathfrak{L}(H)}^{2}\right] \leq M_{1}
$$

for some $M_{1}>0$.

(H2) $B$ is a bounded linear operator on $H$ with operator bound $M_{B}>0$.

(H3) $f_{1}:[0, T] \times H \rightarrow H$ and $f_{2}:[0, T] \times H \times \wp_{\lambda^{2}}(H) \rightarrow H, f_{4}:[0, T] \times H \times U \rightarrow H$ are $\mathfrak{F}_{t}$-measurable mappings such that

(a) $\exists K_{1}:[0, \infty) \times[0, \infty) \rightarrow[0, \infty)$ such that

i. $K_{1}(\cdot, \cdot \cdot)$ is continuous, monotone nondecreasing, and concave.

ii. $\left\|f_{1}(t, y)\right\|^{2}+\left\|f_{2}(t, y, v)\right\|^{2}+\|B(y)\|^{2} \leq K_{1}\left(\|y\|^{2},\|v\|_{\lambda^{2}}^{2}\right)$, for all $t \in[0, T], y \in H, v \in \wp_{\lambda^{2}}(H)$. 
(b) $\exists K_{2}:[0, \infty) \rightarrow[0, \infty)$ such that

i. $K_{2}(\cdot)$ is continuous, monotone nondecreasing, concave, and $K_{2}(0)=0$.

ii. $\left\|f_{1}\left(t, y_{1}\right)-f_{1}\left(t, y_{2}\right)\right\|^{2}+\left\|f_{2}\left(t, y_{1}, v_{1}\right)-f_{2}\left(t, y_{2}, v_{2}\right)\right\|^{2}+\left\|B\left(y_{1}\right)-B\left(y_{2}\right)\right\| \leq K_{2}\left(\left\|y_{1}-y_{2}\right\|^{2}\right)+$ $M_{K_{2}} \rho^{2}\left(v_{1}, v_{2}\right)$, for some $M_{K_{2}}>0$ and for all $t \in[0, T], y_{1}, y_{2} \in H, v_{1}, v_{2} \in \wp_{\lambda^{2}}(H)$.

iii. For all $s \in[0, T], x(s), y(s) \in H$,

$$
\begin{aligned}
\left(\int_{0}^{t} \int_{Z}\left\|f_{4}(s, x(s-), z)-f_{4}(s, y(s-), z)\right\|^{2} v(d z) d s\right) \vee & \\
& \left(\int_{0}^{t} \int_{Z}\left\|f_{4}(s, x(s-), z)-f_{4}(s, y(s-), z)\right\|^{4} v(d z) d s\right)^{\frac{1}{2}} \leq \int_{0}^{t} K_{2}\left(\|x(s)-y(s)\|^{2}\right) d s
\end{aligned}
$$

and

$$
\left(\int_{0}^{t} \int_{Z}\left\|f_{4}(s, x(s-), z)\right\|^{2} v(d z) d s\right) \vee\left(\int_{0}^{t} \int_{Z}\left\|f_{4}(t, x(s-), z)\right\|^{4} v(d z) d s\right)^{\frac{1}{2}} \leq \int_{0}^{t} K_{2}\left(\|x(s)\|^{2}\right) d s
$$

(c) If a nonnegative, continuous function $W(t)$ satisfies $W(0)=0$ and

$$
W(t) \leq \delta \int_{0}^{t} K_{2}(W(s)) d s
$$

for all $0 \leq t \leq T$, where $\delta>0$, then $W(t)=0$, for all $0 \leq t \leq T$.

(d) For all fixed $T>0, \xi>0$ and $y \geq 0$, the initial value problem

$$
\left\{\begin{array}{l}
u^{\prime}(t)=\xi K_{1}(u(t), y), t \in[0, T] \\
u(0)=u_{0} \geq 0
\end{array}\right.
$$

has a global solution on $[0, T]$.

(H4) $f_{3}:[0, T] \rightarrow \mathfrak{L}(K, H)$ is a bounded, strongly measurable mapping;

(H5) $\left\{\beta^{h}(t): t \geq 0\right\}$ is a $K$-valued fractional Brownian motion with Hurst parameter $h \in\left(\frac{1}{2}, 1\right)$;

(H6) $\tilde{N}(d t, d \eta)$ is a compensating Poisson random measure induced by a Poisson point process $p(\cdot)$, which is independent of the $\operatorname{fBm}\left\{\beta^{h}(t): t \geq 0\right\}$ and takes values in a measurable space $(Z, \mathfrak{B}(Z))$.

(H7) $x_{0}, x_{1} \in L_{0}^{2}(\Omega ; H)$ are both $\left(\mathfrak{F}_{0}, \mathfrak{B}(H)\right)$-measurable and independent of $\left\{\beta^{h}(t): t \geq 0\right\}$ and the Poisson point process $p(\cdot)$.

The following technical properties involving stochastic integrals under assumptions (H1), (H4), and (H5) are used in the proofs of the main results in this paper.

Lemma 3.2. Assume (H1), (H4), and (H5). Then,

(i) $E\left\|\int_{0}^{t} S(t-s) f_{3}(s) d \beta^{h}(s)\right\|^{2} \leq \bar{M}^{2} L^{2} t^{2 h} \sum_{j=1}^{\infty} v_{j}$

(ii) $\lim _{h \rightarrow 0^{+}} E\left\|\int_{0}^{t}[S(t+h-s)-S(t-s)] f_{3}(s) d \beta^{h}(s)\right\|^{2}=0$

(iii) There exists a positive constant $\tilde{C}$ such that

$$
\begin{aligned}
E\left\|\int_{0}^{t} S(t-s) \int_{Z} f_{4}(s, x(s-), z) \tilde{N}(d s, d \eta)\right\|^{2} & \leq \tilde{C}\left[E \int_{0}^{t} \int_{Z}\left\|f_{4}(s, x(s-), z)\right\|^{2} d z d s\right. \\
& \left.+E\left(\int_{0}^{t} \int_{Z}\left\|f_{4}(s, x(s-), z)\right\|^{4} d z d s\right)^{\frac{1}{2}}\right]
\end{aligned}
$$


for all $0 \leq t \leq T$, where $L$ is defined in (2.2) and $\left\{v_{j}: j \in \mathbb{N}\right\}$ in the discussion leading to Definition 2.1.

Proof. Since $T$ is fixed, property (i) can be established as in Lemma 6 in [3] by using the uniform bound $\bar{M}$ instead of the estimate $\|S(t)\| \leq M_{A} e^{\alpha|t|}$ (cf. Proposition 2.5).

Property (ii) can be established as in Lemma 6 in [3] by replacing $S(t-s)$ by $[S(t+h-s)-S(t-s)]$ and replacing $\|S(t)\|_{\mathfrak{B} \mathfrak{L}(H)} \leq M_{A} e^{\alpha|t|}$ by

$$
\|S(t+h-s)-S(t-s)\|_{\mathfrak{B} \mathfrak{L}(H)} \leq N_{S}|h|
$$

(cf. Proposition 2.5) to yield

$$
0 \leq E\left\|\int_{0}^{t}[S(t+h-s)-S(t-s)] f_{3}(s) d \beta^{h}(s)\right\|^{2} \leq N_{S}^{2}|h|^{2} T^{2 h} \sum_{j=1}^{\infty} v_{j},
$$

for all $0 \leq t \leq T$. Letting $h \rightarrow 0^{+}$in (3.2) completes the proof of Lemma 3.2.

Property (iii) can be established as in Lemma 13 of [29] by applying the Burkholder-Davis Gundy inequality, and this completes the proof of the lemma.

Theorem 3.3. Assume (H1)-(H7). Then, there exists a unique mild solution of (1.2) in $X_{T, 2}$ with probability law in $\mathscr{C}_{\lambda^{2}}$.

We return to the proof of Theorem 3.3 after discussing several supporting propositions. Unless otherwise stated, we assume (H1)-(H8) throughout the rest of the manuscript. Let $\mu_{0} \in \mathscr{C}_{\lambda^{2}}$ be fixed, and define sequences of recursive approximations as follows:

$$
\begin{aligned}
x_{0}(t) & =(C(t)-S(t) B) x_{0}+S(t)\left(x_{1}-f_{1}\left(0, x_{0}\right)\right), 0 \leq t \leq T, \\
x_{n}(t) & =x_{0}(t)+\int_{0}^{t} C(t-s)\left[B x_{n-1}(s)+f_{1}\left(s, x_{n-1}(s)\right)\right] d s \\
& +\int_{0}^{t} S(t-s) f_{2}\left(s, x_{n-1}(s), \mu_{0}(s)\right) d s+\int_{0}^{t} S(t-s) f_{3}(s) d \beta^{h}(s) \\
& +\int_{0}^{t} S(t-s) \int_{Z} f_{4}\left(s, x_{n-1}(s-), \eta\right) \tilde{N}(d s, d \eta), 0 \leq t \leq T, n \geq 1 .
\end{aligned}
$$

Proposition 3.4. $x_{n} \in X_{T, 2}$ for all $n \geq 0$, and there exist positive constants $\mathcal{C}_{1}, \mathcal{C}_{2}, \mathcal{C}_{3}$ (independent of $n, m$ ) such that for all $n, m \geq 1$ and $0 \leq t \leq T$,

$$
\begin{aligned}
& \text { (i) } E\left\|x_{n}(t)\right\|^{2} \leq \mathcal{C}_{1}+\mathcal{C}_{2} \int_{0}^{t} K_{1}\left(E\left\|x_{n-1}(s)\right\|^{2}, \sup _{0 \leq s \leq t}\left\|\mu_{0}(s)\right\|_{\lambda^{2}}^{2}\right) d s \\
& \text { (ii) } E\left\|x_{n}(t)-x_{m}(t)\right\|^{2} \leq \mathcal{C}_{3} \int_{0}^{t} K_{2}\left(E\left\|x_{n-1}(s)-x_{m-1}(s)\right\|^{2}\right) d s .
\end{aligned}
$$

Proof. Clearly, $x_{0} \in X_{T, 2}$. By induction and Lemma 3.2 (ii) and (ii) (see [2, 18, 29]), $x_{n} \in X_{T, 2}$. Let $n \geq 1$. Applying Jensen and Hölder inequalities and Lemma 3.2 (i) and (ii), along with iterated calculations including 
the hypotheses, yield

$$
\begin{aligned}
E\left\|x_{n}(t)\right\|^{2} & \leq 6 E\left\|(C(t)-S(t) B) x_{0}\right\|^{2}+6 E\left\|S(t)\left(x_{1}-f_{1}\left(0, x_{0}\right)\right)\right\|^{2} \\
& +6 E \| \int_{0}^{t} C(t-s)\left[B x_{n-1}(s)+f_{1}\left(s, x_{n-1}(s)\right] d s \|^{2}\right. \\
& +6 E\left\|\int_{0}^{t} S(t-s) f_{2}\left(s, x_{n-1}(s), \mu_{0}(s)\right) d s\right\|^{2} \\
& +6 E\left\|\int_{0}^{t} S(t-s) f_{3}(s) d \beta^{h}(s)\right\|^{2} \\
& +6 E\left\|\int_{0}^{t} S(t-s) \int f_{Z}\left(s, x_{n-1}(s-), \eta\right) \tilde{N}(d s, d \eta)\right\|^{2} \\
& \leq 6\left[M_{1}^{2}\left(1+M_{B}^{2}\right) E\left\|x_{0}\right\|^{2}+2 M_{1}^{2}\left(E\left\|x_{1}\right\|^{2}+E\left\|f_{1}\left(0, x_{0}\right)\right\|^{2}\right)\right] \\
& +6\left[\bar{M}^{2} L^{2} T^{2 h} \sum_{j=1}^{\infty} v_{j}+2 M_{1}^{2} T \int_{0}^{t} E\left(\left\|B x_{n-1}(s)\right\|^{2}+\| f_{1}\left(s, x_{n-1}(s) \|^{2}\right)\right]\right. \\
& +6 M_{1}^{2} T \int_{0}^{t} E\left\|f_{2}\left(s, x_{n-1}(s), \mu_{0}(s)\right)\right\|^{2} d s \\
& +\mathcal{C}_{1}+\left(12 M_{1}^{2} T+6 \tilde{C}\right) \int_{0}^{t} K_{1}\left(E\left\|x_{n-1}(s)\right\|^{2}, \operatorname{esp}_{0 \leq s \leq t}\left\|\mu_{0}(s)\right\|_{\lambda^{2}}^{2}\right) d s \\
& +6 \tilde{C} E \int_{0}^{t} \int_{Z}^{t}\left\|f_{4}\left(s, x_{n-1}(s-), z\right)\right\|^{2} v(d z) d s \\
& +6 \tilde{C} E \int_{0}^{t}\left(\int_{Z}\left\|f_{4}\left(s, x_{n-1}(s-), z\right) v(d z)\right\|^{4}\right)^{\frac{1}{2}} d s \\
& \\
& \\
& \\
&
\end{aligned}
$$

for all $0 \leq t \leq T$, and where $\mathcal{C}_{1}, \mathcal{C}_{2}$ are defined as follows:

$$
\begin{aligned}
& \mathcal{C}_{1}=6\left[M_{1}^{2}\left(1+M_{B}^{2}\right) E\left\|x_{0}\right\|^{2}+2 M_{1}^{2}\left(E\left\|x_{1}\right\|^{2}+E\left\|f_{1}\left(0, x_{0}\right)\right\|^{2}\right)+\bar{M}^{2} L^{2} T^{2 h} \sum_{j=1}^{\infty} v_{j}\right], \\
& \mathcal{C}_{2}=12 M_{1}^{2} T+6 \tilde{C} .
\end{aligned}
$$


Thus, (i) is proved. Similarly for (ii), let $m, n \geq 1$ and $\mathrm{C}_{3}=3\left(2 M_{1}^{2} T+M_{1}^{2} T+\tilde{C}\right)$ to yield

$$
\begin{aligned}
E\left\|x_{n}(t)-x_{m}(t)\right\|^{2} & \leq 6 M_{1}^{2} T \int_{0}^{t} E\left(\left\|B x_{n-1}(s)-B x_{m-1}(s)\right\|^{2}\right. \\
& +\| f_{1}\left(s, x_{n-1}(s)\right)-f_{1}\left(s, x_{m-1}(s) \|^{2}\right) d s \\
& +3 M_{1}^{2} T \int_{0}^{t} E\left\|f_{2}\left(s, x_{n-1}(s), \mu_{0}(s)\right)-f_{2}\left(s, x_{m-1}(s), \mu_{0}(s)\right)\right\|^{2} d s \\
& +3 \tilde{C} E \int_{0}^{t}\left[\int_{Z}\left\|f_{4}\left(s, x_{n-1}(s-), z\right)-f_{4}\left(s, x_{m-1}(s), z\right)\right\|^{2} v(d z)\right. \\
& \left.\left.+\int_{Z}\left\|f_{4}\left(s, x_{n-1}(s-), z\right)-f_{4}\left(s, x_{m-1}(s), z\right)\right\|^{2} v(d z)\right)^{\frac{1}{2}}\right] d s \\
& \leq 3\left(2 M_{1}^{2} T+M_{1}^{2} T \tilde{C}\right) \int_{0}^{t} K_{2}\left(E\left\|x_{n-1}(s)-x_{m-1}(s)\right\|^{2}\right) d s \\
& =\mathcal{C}_{3} \int_{0}^{t} K_{2}\left(E\left\|x_{n-1}(s)-x_{m-1}(s)\right\|^{2}\right) d s,
\end{aligned}
$$

for all $0 \leq t \leq T$, and that completes the proof of (ii).

Proposition 3.5. There exists an integrable function $u:[0, T] \rightarrow \mathbb{R}$ such that

$$
E\left\|x_{n}(t)\right\|^{2} \leq u(t)
$$

for all $n \geq 0$ and for all $0 \leq t \leq T$.

Proof. Let $u(t)$ be the global solution of (3.1) where the initial condition is chosen to be the greater than $\max \left\{\mathcal{C}_{1}\right.$, sup $\left.E\left\|x_{0}(t)\right\|^{2}\right\}$. We show (3.5) using induction. For $n=0$, (3.5) holds by definition of $u(\cdot)$ (with $\left.\xi=e_{2}\right)$. Indeed, observe that

$$
\begin{aligned}
u(t) & =u_{0}+\mathcal{C}_{2} \int_{0}^{t} K_{1}\left(u(s), \sup _{0 \leq s \leq T}\|\mu(s)\|_{\lambda^{2}}^{2}\right) d s \\
& \geq \max \left\{\mathcal{C}_{1}, \sup _{0 \leq t \leq T} E\left\|x_{0}(t)\right\|^{2}\right\}+\varrho_{2} \int_{0}^{t} K_{1}\left(u(s), \sup _{0 \leq s \leq T}\left\|\mu_{(}(s)\right\|_{\lambda^{2}}^{2}\right) d s \\
& \geq E\left\|x_{0}(t)\right\|^{2}
\end{aligned}
$$

Next, let $n \geq 1$ be fixed and assume that

$$
E\left\|x_{n-1}(t)\right\|^{2} \leq u(t), \text { for all } 0 \leq t \leq T .
$$

Using (3.1), 3.4 (i), and (H3), it follows

$$
0 \leq \mathcal{C}_{2} \int_{0}^{t}\left[K_{1}\left(u(s), \sup _{0 \leq s \leq T}\left\|\mu_{0}(s)\right\|_{\lambda^{2}}^{2}\right)-K_{1}\left(E\left\|x_{n-1}(s)\right\|^{2}, \sup _{0 \leq s \leq T}\left\|\mu_{0}(s)\right\|_{\lambda^{2}}^{2}\right)\right] d s \leq u(t)-E\left\|x_{n}(t)\right\|^{2}
$$

by induction, and thus, the proposition is proved. 
Proposition 3.6. $\left\{x_{n} \mid n \geq 1\right\}$ is a Cauchy Sequence in $X_{T, 2}$.

Proof. For $n \geq 1$, define the function $\Psi_{n}:[0, T] \rightarrow \mathbb{R}$ by

$$
\Psi_{n}(t)=\sup _{m \geq n} E\left\|x_{m+n}(t)-x_{n}(t)\right\|^{2} .
$$

Each function $\Psi_{n}$ is well-defined, uniformly bounded, and monotone nondecreasing in $t$. Since for every $0 \leq t \leq T,\left\{\Psi_{n}(t): n \geq 1\right\}$ is a monotone, nonincreasing sequence, we can define a monotone, nonincreasing function $\Psi:[0, T] \rightarrow \mathbb{R}$ by

$$
\Psi(t)=\lim _{n \rightarrow \infty} \Psi_{n}(t)
$$

Using Proposition 3.4 (ii) and (H3), it follows that for every $n \geq 1$,

$$
\Psi(t) \leq \Psi_{n}(t) \leq \mathcal{C}_{3} \int_{0}^{t} K_{2}\left(\Psi_{n}(s)\right) d s, 0 \leq t \leq T .
$$

Applying the Lebesgue dominated convergence theorem with (3.7) and (3.8) subsequently yields

$$
\Psi(t) \leq \mathcal{C}_{3} \int_{0}^{t} K_{2}(\Psi(s)) d s .
$$

Since $\sup _{0 \leq t \leq T} E\left\|x_{m+n}(t)-x_{n}(t)\right\|^{2} \leq \Psi_{n}(t)$ and $\lim _{n \rightarrow \infty} \Psi_{n}(T)=\Psi(T)=0$, it follows that $\sup _{0 \leq t \leq T} E\left\|x_{m+n}(t)-x_{n}(t)\right\|^{2}=0$. Hence, $\left\{x_{n} \mid n \geq 1\right\}$ is a Cauchy Sequence in $X_{T, 2}$, as desired.

Proposition 3.7. Let $\mu \in \mathscr{C}_{\lambda^{2}}$. Then IVP (1.2) has a unique global parametrized mild solution in $X_{T, 2}$.

Proof. Let $\mu \in \mathscr{C}_{\lambda^{2}}$ be fixed. Using Proposition 3.6, the completeness $X_{T, 2}$ guarantees there exists a stochastic process $x$ such that

$$
\lim _{n \rightarrow \infty} \sup _{0 \leq t \leq T} E\left\|x_{n}(t)-x(t)\right\|^{2}=0
$$

Furthermore, from (H3)

$$
\lim _{n \rightarrow \infty} K_{2}\left(\sup _{0 \leq t \leq T} E\left\|x_{n}(t)-x(t)\right\|^{2}\right)=K_{2}(0)=0 .
$$

As such, taking the limit as $n \rightarrow \infty$ (3.4) shows that $x(t)$ itself satisfies (3.1) for all $0 \leq t \leq T$. Hence, $x$ is a mild solution of (1.2) on $[0, T]$.

Suppose $x, y \in X_{T, 2}$ are two mild solutions of (1.2). Then, arguing as in Proposition 3.4 (ii), it can be shown that for all $0 \leq t \leq T$,

$$
\sup _{0 \leq t \leq T} E\|x(t)-y(t)\|^{2} \leq \mathcal{C}_{3} \int_{0}^{t} K_{2}\left(\sup _{0 \leq \tau \leq s} E\|x(\tau)-y(\tau)\|^{2}\right) d s,
$$

and so (H3) (ii) (c) implies that $\sup _{0 \leq t \leq T} E\|x(t)-y(t)\|^{2}=0$. Thus, $x=y$ in $X_{T, 2}$, guaranteeing uniqueness, for a fixed $\mu \in \mathscr{C}_{\lambda^{2}}$, and this completes the proof.

\section{Proof of Theorem 3.3}

We modify arguments in [20] and [13] to conclude there exists an unique probability measure $\mu \in \mathscr{C}_{\lambda^{2}}$, the probability law of $x(t)$, that solves (1.2). To this end, let $x_{v}$ denote the unique parametrized mild solution to (1.2) guaranteed by Proposition 3.6 for the fixed probability law $v \in \mathscr{C}_{\lambda^{2}}$, and let $\mathfrak{L}\left(x_{v}\right)=\left\{\mathfrak{L}\left(x_{\nu}(t)\right): t \in[0, T]\right\}$ be the probability law of $x_{v}$. As in [13], we use the stronger uniform metric $D_{T}$ on $D\left([0, T] ;\left(\wp \lambda^{2}(H), \rho\right)\right)$ to avoid 
some complications, but we will appeal to the complete Skorohod metric in final step of the Cauchy sequence argument.

Let $\mu, v \in \mathscr{C}_{\lambda^{2}}$. Note that since $x_{\mu} \in X_{T, 2}, \mathfrak{L}\left(x_{\mu}(t)\right) \in \wp_{\lambda^{2}}(H)$, for any $t \in[0, T]$. Next, we must show that $t \mapsto \mathfrak{L}\left(x_{\mu}(t)\right)$ is right-continuous with finite left-limits. Let $c \in[0, T)$ and $h>0$ be sufficiently small. Then the right-continuity of $x_{\mu}$ and continuity of $K_{1}, K_{2}$ ensures that

$$
\lim _{h \rightarrow 0} E\left\|x_{\mu}(c+h)-x_{\mu}(c)\right\|^{2}=0, \text { for all } 0 \leq c<T .
$$

Next, for all $0 \leq c<T$ and $\phi \in C_{\lambda^{2}}(H)$, the definition of the metric $\rho$ yields

$$
\mid\left\langle\phi, \mathfrak{L}\left(x_{\mu}(c+h)\right)-\mathfrak{L}\left(x_{\mu}(c)\right)\right\rangle \leq\|\phi\|_{C_{\lambda^{2}}} E\left\|x_{\mu}(c+h)-x_{\mu}(c)\right\|,
$$

so, we may conclude that

$$
\lim _{h \rightarrow 0} \rho\left(\mathfrak{L}\left(x_{\mu}(c+h)\right), \mathfrak{L}\left(x_{\mu}(c)\right)\right)=\lim _{h \rightarrow 0} \sup _{\|\phi\|_{C_{\lambda^{2}} \leq 1}} \int_{H} \phi\left(\mathfrak{L}\left(x_{\mu}(c+h)\right)-\mathfrak{L}\left(x_{\mu}(c)\right)\right)=0 .
$$

A similar argument can made to conclude finite left-limits.

Using (H3), in conjunction with the technique used to establish Proposition 3.4, we arrive at

$$
E\left\|x_{\mu}(t)-x_{v}(t)\right\|^{2} \leq \mathscr{C}_{3} \int_{0}^{t}\left[K_{2}\left(E\left\|x_{\mu}(s)-x_{v}(s)\right\|^{2}\right)+M_{K_{2}}^{2} \rho^{2}(\mu(s), v(s))\right] d s .
$$

Since $K_{2}$ is concave on $[0, \infty)$, there exists positive constants $a$ and $b$ such that $K_{2}(u) \leq a u+b$. So,

$$
\begin{aligned}
E \| x_{\mu}(t) & -x_{v}(t) \|^{2} \leq \mathscr{C}_{3} \int_{0}^{t}\left[a E\left\|x_{\mu}(s)-x_{v}(s)\right\|^{2}+b+M_{K_{2}}^{2} \rho^{2}(\mu(s), v(s))\right] d s \\
& \leq \mathscr{C}_{3} \int_{0}^{t}\left[b+M_{K_{2}}^{2} \rho^{2}(\mu(s), v(s))\right] d s+a \mathscr{C}_{3} \int_{0}^{t} E\left\|x_{\mu}(s)-x_{\nu}(s)\right\|^{2} d u,
\end{aligned}
$$

from which an application of Gronwall's inequality yields

$$
\begin{aligned}
E\left\|x_{\mu}(t)-x_{v}(t)\right\|^{2} & \leq \mathscr{C}_{3} \int_{0}^{t}\left[b+M_{K_{2}}^{2} \rho^{2}(\mu(s), v(s))\right] d s \exp \left(a \mathscr{C}_{3} t\right) \\
& \leq \mathscr{C}_{3} t \exp \left(a \mathscr{C}_{3} t\right)\left[b+M_{K_{2}}^{2} D_{T}^{2}(\mu, v)\right] .
\end{aligned}
$$

For sufficiently small $t>0$, we have

$$
\mathscr{C}_{3} t \exp \left(a \mathscr{C}_{3} t\right)\left[b+M_{K_{2}}^{2} D_{T}^{2}(\mu, v)\right] \leq C D_{T}^{2}(\mu, v),
$$

for some $0<C<1$. Therefore, there exists a $T_{1} \in(0, T]$ such that

$$
E\left\|x_{\mu}(t)-x_{v}(t)\right\|^{2} \leq C D_{T_{1}}^{2}(\mu, v), \text { for all } t \in\left[0, T_{1}\right] .
$$

Hence,

$$
D_{T_{1}}^{2}\left(\mathfrak{L}\left(x_{\mu}\right), \mathfrak{L}\left(x_{v}\right)\right) \leq E\left\|x_{\mu}(t)-x_{v}(t)\right\|^{2} \leq C D_{T_{1}}^{2}(\mu, v),
$$

for all $t \in\left[0, T_{1}\right]$.

Let $\mu_{0} \in D\left(\left[0, T_{1}\right] ;\left(\wp_{\lambda^{2}}(H), \rho\right)\right)$ be fixed, and define a recursive sequence in $D\left(\left[0, T_{1}\right] ;\left(\wp_{\lambda^{2}}(H), \rho\right)\right)$ as follows:

$$
\begin{aligned}
& \mu_{1}=\mathfrak{L}\left(x_{\mu_{0}}\right), \\
& \mu_{n}=\mathfrak{L}\left(x_{\mu_{n-1}}\right), n \geq 2 .
\end{aligned}
$$


Although the uniform metric $D_{T_{1}}$ on $D\left(\left[0, T_{1}\right] ;\left(\wp_{\lambda^{2}}(H), \rho\right)\right)$ is not separable, (3.10) shows that $\left\{\mu_{n}: n \geq 1\right\}$ Cauchy sequence for $D_{T_{1}}$. Thus, for the complete Skorohod metric, $S_{T_{1}}, \mu_{n} \rightarrow \mu_{\infty} \in D\left(\left[0, T_{1}\right] ;\left(\wp_{\lambda^{2}}(H)\right.\right.$, $\left.\left.\rho\right)\right)$, as $n \rightarrow \infty$. Arguing as in (3.10) yields

$$
S_{T_{1}}\left(\mathfrak{L}\left(x_{\mu_{n-1}}\right), \mathfrak{L}\left(x_{\mu_{\infty}}\right)\right) \leq S_{T_{1}}\left(\mu_{n-1}, \mu_{\infty}\right),
$$

for which an application of triangle inequality yields $\mathfrak{L}\left(x_{\mu_{\infty}}\right)=\mu_{\infty}$. By iteration, the existence and uniqueness on $[0, T]$ can be obtained, and this completes the proof.

Remark 3.8. We recover the existence and uniqueness of a mild solution of (1.2) under the classical Lipschitz condition as a special case of Theorem 3.3. Furthermore, the dependence of the nonlinearities on the probability law and the range of the Hurst parameter enables us to generalize and/or view the existence results in $[13,20]$ as corollaries of Theorem 3.3 .

\section{Stability in Mean Square}

We further restrict the function $K_{2}$ in (H3)(b) and the growth condition only on $f_{1}$ in (H3)(ii)(b) as follows:

$(\mathrm{H} 8) K_{2}(s)>0$ for $s>0$ and $\int_{0^{+}} \frac{d s}{K_{2}(s)}=\infty$.

(H9) $\exists M_{f_{1}}>0$ such that for all $t \in[0, T], y_{1}, y_{2} \in H$,

$$
\left\|f_{1}\left(t, y_{1}\right)-f_{1}\left(t, y_{2}\right)\right\|^{2} \leq M_{f_{1}}\left\|y_{1}-y_{2}\right\|^{2} .
$$

Remark 4.1. As stated in Remark 8 of [29], there are several examples of functions $K_{2}$ which are natural growth conditions to impose on the nonlinearities and which satisfy (H3) and (H8).

Definition 4.2. A mild solution $x$ of (1.2) on [0,T] with initial condition $\left(x_{0}, x_{1}\right)$ is stable in mean square if $\forall \epsilon>0, \exists \delta>0$ such that

$$
E\left\|x_{0}-\bar{x}_{0}\right\|^{2}+E\left\|x_{1}-\bar{x}_{1}\right\|^{2}<\delta
$$

implies that

$$
E\left(\sup _{0 \leq s \leq T}\|x(s)-\bar{x}(s)\|^{2}\right) \leq \epsilon,
$$

where $\bar{x}$ is the solution to (1.2) with initial condition $\left(\bar{x}_{0}, \bar{x}_{1}\right)$.

Theorem 4.3. Let $x$ and $\bar{x}$ be solutions on $[0, T]$ of (1.2) with initial conditions $\left(x_{0}, x_{1}\right)$ and $\left(\bar{x}_{0}, \bar{x}_{1}\right)$, respectively. Assume (H1)-(H7) as in Theorem 3.3, (H8), and $f_{1}$ satisfies (H9) instead of (H3)(ii)(b), then the mild solution of (1.2) is stable in mean square.

Proof. Let $\epsilon>0$, and let $x$ and $\bar{x}$ with probability laws $\mu_{x}$ and $\mu_{\bar{x}}$, respectively, be solutions on $[0, T]$ of (1.2) with initial conditions $\left(x_{0}, x_{1}\right)$ and $\left(\bar{x}_{0}, \bar{x}_{1}\right)$, respectively. Subtracting the variation of parameters formulation of $\bar{x}$ from $x$ yields

$$
\begin{aligned}
x(s)-\bar{x}(s) & =(C(s)-S(s) B)\left(x_{0}-\bar{x}_{0}\right)+S(s)\left[\left(x_{1}-\bar{x}_{1}\right)-\left(f_{1}\left(0, x_{0}\right)-f_{1}\left(0, \bar{x}_{0}\right)\right)\right] \\
& +\int_{0}^{s} C(s-\tau)\left[B(x(\tau)-\bar{x}(\tau))+\left(f_{1}(\tau, x(\tau))-f_{1}(\tau, \bar{x}(\tau))\right)\right] d \tau \\
& +\int_{0}^{s} S(s-\tau)\left[f_{2}\left(\tau, x(\tau), \mu_{x}(\tau)\right)-f_{2}\left(\tau, \bar{x}(\tau), \mu_{\bar{x}}(\tau)\right)\right] d \tau \\
& +\int_{0}^{s} S(s-\tau) \int_{Z}\left[f_{4}(\tau, x(\tau-), \eta)-f_{4}(\tau, \bar{x}(\tau-), \eta)\right] \tilde{N}(d \tau, d \eta)
\end{aligned}
$$


Using calculations similar to those used to prove Proposition 3.4 and Theorem 3.3 yield

$$
\begin{aligned}
E \sup _{0 \leq s \leq t}\|x(s)-\bar{x}(s)\|^{2} & \leq 6\left(M_{1} E\left\|x_{0}-\bar{x}_{0}\right\|^{2}+M_{1} E\left\|x_{1}-\bar{x}_{1}\right\|^{2}\right)+M^{\star} \int_{0}^{t}\left[K_{2}\left(E \sup _{0 \leq r \leq s}\|x(r)-\bar{x}(r)\|^{2}\right)\right. \\
& \left.+E \sup _{0 \leq r \leq s}\|x(r)-\bar{x}(r)\|^{2}\right] d s, \forall t \in[0, T],
\end{aligned}
$$

where $M^{\star}$ is a positive constant involving $\mathscr{C}_{3}, T$, and $M_{1}$. Let

$$
\bar{K}(u)=M^{\star}\left(K_{2}(u)+u\right) .
$$

Clearly, (H3) implies $\bar{K}$ is concave, nondecreasing, continuous from $[0, \infty)$ to $[0, \infty), \bar{K}(0)=0, K_{2}(u) \geq \bar{K}(u)$ for all $0 \leq u \leq 1$, and $\int_{0^{+}} \frac{d u}{\bar{K}(u)}=\infty$. As such, for $\bar{\epsilon}=\frac{1}{2} \epsilon, \lim _{\tau \rightarrow 0^{+}} \int_{\tau}^{\bar{\epsilon}} \frac{d u}{\bar{K}(u)}=\infty$. Hence, $\exists 0<\delta<\bar{\epsilon}$ for which $\int_{\delta}^{\bar{\epsilon}} \frac{d u}{\bar{K}(u)} \geq T$. Let $u_{0}=6\left(M_{1} E\left\|x_{0}-\bar{x}_{0}\right\|^{2}+M_{1} E\left\|x_{1}-\bar{x}_{1}\right\|^{2}\right), u(t)=E \sup _{0 \leq s \leq t}\|x(s)-\bar{x}(s)\|^{2}$, and $v(t)=1$. Thus,

when $u_{0} \leq \delta \leq \bar{\epsilon}$, we have $\int_{u_{0}}^{\bar{\epsilon}} \frac{d u}{\bar{K}(u)} \geq \int_{\delta}^{\bar{\epsilon}} \frac{d u}{\bar{K}(u)} \geq T=\int_{0}^{T} v(s) d s$. So, by Lemma $2.8, u(t) \leq \epsilon$ for any $t \in[0, T]$, and the proof of the theorem is complete.

\section{Example}

To illustrate the applicability of the theory we return to the motivating example and impose the following conditions:

(A1) $a_{j k}: \mathscr{D} \rightarrow \mathbb{R}(1 \leq j, k \leq n)$ and $C: \mathscr{D} \rightarrow \mathbb{R}$ are continuous on $\overline{\mathscr{D}}$ and are defined to ensure that the Gärding inequality is satisfied. (See [7] for sufficient conditions to guarantee that this condition holds.)

$(\mathrm{A} 2) \mathfrak{B}: L^{2}(\mathscr{D}) \rightarrow \mathfrak{B}$ is a bounded, linear operator with operator bound $M_{\mathfrak{B}}$.

(A3) $F_{1}$ satisfies the Caratheodory conditions (i.e., measurable in $(t, z)$ and continuous in the third variable) such that

1. $\left\|F_{2}^{1}(t, z, y)\right\|_{L^{2}(\mathscr{D})}^{2} \leq \kappa_{1}\left(\|y\|_{L^{2}(\mathscr{D})}^{2}\right)$ for all $0 \leq t \leq T, z \in \mathscr{D}, y \in \mathbb{R}$ and for some continuous, monotone nondecreasing and concave function $\kappa_{1}(\cdot)$ such that

$$
\left\{\begin{array}{l}
u^{\prime}(t)=\xi \kappa_{1}(u(t)), t \in[0, T] \\
u(0)=u_{0} \geq 0
\end{array}\right.
$$

has a global solution for all fixed $T, \xi>0$,

2. $\left\|F_{2}^{1}\left(t, z, y_{1}\right)-F_{2}^{1}\left(t, z, y_{2}\right)\right\|_{L^{2}(\mathscr{D})}^{2} \leq \kappa_{2}\left(\left\|y_{1}-y_{2}\right\|_{L^{2}(\mathscr{D})}^{2}\right)$ for all $0 \leq t \leq T, z \in \mathscr{D}, y_{1}, y_{2} \in \mathbb{R}$ and for some $\kappa_{2}(\cdot)$ satisfying $(\mathrm{H} 3)(\mathrm{b})$ and $(\mathrm{H} 8)$;

(A4) $F_{2}^{2}$ satisfies the Caratheodory conditions and

1. $\left\|F_{2}(t, z, y)\right\|_{L^{2}(\mathscr{D})} \leq \bar{M}_{F_{2}}\left[1+\|z\|_{L^{2}(\mathscr{D})}\right]$ for all $0 \leq t \leq T, z \in \mathscr{D}, y \in L^{2}(\mathscr{D})$ and some $\bar{M}_{F_{2}}>0$,

2. $F_{2}(t, z, \cdot): L^{2}(\mathscr{D}) \rightarrow L^{2}(\mathscr{D})$ is in $\mathscr{C}$ for each $0 \leq t \leq T, z \in \mathscr{D}$;

(A5) $g:[0, T] \times \mathscr{D} \rightarrow \mathfrak{L}\left(\mathbb{R}, L^{2}(\mathscr{D})\right)$ is a bounded, strongly measurable function.

(A6) $\int_{U \backslash\{0\}} u^{2} \lambda(d u)<\infty$ and $\int_{U \backslash\{0\}} u^{4} \lambda(d u)<\infty$

(A7) $\xi$ is an $\mathfrak{F}_{0}$-measurable random variable independent of $B^{\mathcal{H}}$ with almost surely continuous paths.

Let $H=K=L^{2}(\mathscr{D})$ and define the operator $A: H \rightarrow H$ by

$$
A x(t, \cdot)=\sum_{j, k=1}^{n} \frac{\partial}{\partial z_{j}}\left[a_{j k}(\cdot) \frac{\partial x(t, \cdot)}{\partial z_{j}}\right]+C(\cdot) x(t, \cdot)
$$


Using (A1), it follows that $A$ is a uniformly elliptic, densely defined, symmetric, self-adjoint operator that generates a strongly continuous cosine family on $H$ (see [5, 26]). Next, define the maps $f_{2}:[0, T] \times H \times \wp_{\lambda^{2}}(H) \rightarrow$ $H, f_{3}:[0, T] \rightarrow \mathfrak{L}(K, H), f_{4}:[0, T] \times H \times Z \rightarrow H$, and $B: H \rightarrow H$, respectively, by

$$
\begin{aligned}
f_{2}(t, x(t), \mu(t))(z) & =F_{2}^{1}(t, z, x(t, z))+\int_{L^{2}(\mathscr{D})} F_{2}^{2}(t, z, y) \mu(t, z)(d y) \\
f_{3}(t)(z) & =F_{3}(t, z) \\
f_{4}(t, x(t-), u)(z) & =x(t-, z) u \\
B\left(x^{\prime}(t)\right)(z) & =\mathfrak{B}\left(\frac{\partial x(t, z)}{\partial t}\right) \\
x(0)(z) & =\xi_{1}(z) \\
x^{\prime}(0)(z) & =\xi_{2}(z)
\end{aligned}
$$

for all $0 \leq t \leq T$ and $z \in \mathscr{D}$. Further, identifying $f_{1}=0$ we see that (1.1) can be written in the abstract form of (1.2). Clearly, (H1)-(H3) and (H5)-(H8) are satisfied. We now show that $f_{2}, f_{3}$ and $B$ satisfies (H4). To this end, observe that using (A4)(1) together with Hölder inequality, we observe that

$$
\begin{aligned}
\left\|\int_{L^{2}(\mathscr{D})} F_{2}(t, \cdot, y) \mu(t, \cdot)(d y)\right\|_{L^{2}(\mathscr{D})} \\
=\left[\int_{\mathscr{D}}\left[\int_{L^{2}(\mathscr{D})} F_{2}(t, z, y) \mu(t, z)(d y)\right]^{2} d z\right]^{\frac{1}{2}} \\
\leq\left[\int_{\mathscr{D}} \int_{L^{2}(\mathscr{D})}\left\|F_{2}(t, z, y)\right\|_{L^{2}(\mathscr{D})}^{2} \mu(t, z)(d y) d z\right]^{\frac{1}{2}} \\
\leq \bar{M}_{F_{2}}\left[\int_{\mathscr{D} L^{2}(\mathscr{D})}\left(1+\|y\|_{L^{2}(\mathscr{D})}\right)_{L^{2}(\mathscr{D})} \mu(t, z)(d y) d z\right]^{\frac{1}{2}} \\
\leq \bar{M}_{F_{2}} \sqrt{m(\mathscr{D})} \sqrt{\|\mu(t)\|_{\lambda^{2}}}, \forall 0 \leq t \leq T, \mu \in \wp_{\lambda^{2}}(H) .
\end{aligned}
$$

(Here, $m$ denotes the Lebesgue measure in $\mathbb{R}^{N}$.) Thus, by (A2), (A3)(1), and (5.2), (H3)(a) is satisfied for $K_{1}$ defined as

$$
K_{1}(a, b)=2\left(\kappa_{1}(a)+M_{\mathfrak{B}}^{2} a+M_{F_{2}}^{2} m(\mathscr{D}) b\right) .
$$

Next, invoking (A4)(2) enables us to see that for all $\mu, v \in \wp_{\lambda^{2}}(H)$,

$$
\begin{aligned}
& \left\|\int_{L^{2}(\mathscr{D})} F_{2}(t, \cdot, y) \mu(t, \cdot)(d y)-\int_{L^{2}(\mathscr{D})} F_{2}(t, \cdot, y) v(t, \cdot)(d y)\right\|_{L^{2}(\mathscr{D})} \\
& \quad=\int_{L^{2}(\mathscr{D})} F_{2}(t, \cdot, y)(\mu(t, \cdot)-v(t, \cdot))(d y) \\
& \quad \leq\|\rho(\mu(t), v(t))\|_{L^{2}(\mathscr{D})} \\
& \quad \leq \sqrt{m(\mathscr{D})} \rho(\mu(t), v(t)), \forall 0 \leq t \leq T .
\end{aligned}
$$

Combining (A2), (A3)(2), and (5.2), we conclude

$$
\begin{aligned}
\left\|f_{2}\left(t, y_{1}, v_{1}\right)-f_{2}\left(t, y_{2}, v_{2}\right)\right\|_{L^{2}(\mathscr{D})}^{2}+ & \left\|B\left(y_{1}\right)-B\left(y_{2}\right)\right\|_{L^{2}(\mathscr{D})}^{2} \\
& \leq 2\left(\kappa_{2}\left(\left\|y_{1}-y_{2}\right\|_{L^{2}(\mathscr{D})}^{2}\right)+M_{\mathfrak{B}}^{2}\left\|y_{1}-y_{2}\right\|_{L^{2}(\mathscr{D})}+m(\mathscr{D}) \rho^{2}\left(v_{1}, v_{2}\right)\right),
\end{aligned}
$$


for all $0 \leq t \leq T ; y_{1}, y_{2} \in L^{2}(\mathscr{D})$; and $v_{1}, v_{2} \in \wp_{\lambda^{2}}\left(L^{2}(\mathscr{D})\right)$. By (A6) and (5.4), (H3)(2) is satisfied. Thus, we can invoke Theorem 3.3 to conclude that (1.1) has a unique mild solution $x \in X_{T, 2}$ with probability law $\{\mu(t): 0 \leq$ $t \leq T\}$. Furthermore, by Theorem 4.3 solutions to (1.1) are stable in mean square.

\section{References}

[1] N. U. Ahmed and X. Ding. A semilinear McKean-Vlasov stochastic evolution equation in Hilbert space. Stochastic Process. Appl., 60(1):65-85, 1995.

[2] S. Albeverio, V. Mandrekar, and B. Rüdiger. Existence of mild solutions for stochastic differential equations and semilinear equations with non-Gaussian Lévy noise. Stochastic Process. Appl., 119(3):835-863, 2009.

[3] V. V. Anh and W. Grecksch. A fractional stochastic evolution equation driven by fractional brownian motion. MCMA, 9(3):189199, 2003.

[4] H. Bergström. Weak convergence of measures. Probability and Mathematical Statistics. Academic Press, Inc., 1982.

[5] A. T. Bharucha-Reid. Random integral equations. Academic Press, New York-London, 1972. Mathematics in Science and Engineering, Vol. 96.

[6] G. Da Prato and J. Zabczyk. Stochastic equations in infinite dimensions, volume 44 of Encyclopedia of Mathematics and its Applications. Cambridge University Press, Cambridge, 1992.

[7] E. B. Davies. Uniformly elliptic operators with measurable coefficients. J. Funct. Anal., 132(1):141-169, 1995.

[8] D. A. Dawson. Critical dynamics and fluctuations for a mean-field model of cooperative behavior. J. Statist. Phys., 31(1):2985, 1983.

[9] D. A. Dawson and J. Gärtner. Large deviations from the McKean-Vlasov limit for weakly interacting diffusions. Stochastics, 20(4):247-308, 1987.

[10] T. E. Duncan, B. Pasik-Duncan, and B. Maslowski. Fractional Brownian motion and stochastic equations in Hilbert spaces. Stoch. Dyn., 2(2):225-250, 2002.

[11] H. O. Fattorini. Second order linear differential equations in Banach spaces, volume 108 of North-Holland Mathematics Studies. North-Holland Publishing Co., Amsterdam, 1985. Notas de Matemática [Mathematical Notes], 99.

[12] J. A. Goldstein. On the convergence and approximation of cosine functions. Aequationes Math., 11:201-205, 1974.

[13] Carl Graham. McKean-Vlasov Itô-Skorohod equations, and nonlinear diffusions with discrete jump sets. Stochastic Process. Appl., 40(1):69-82, 1992.

[14] W. Grecksch and V. V. Anh. A parabolic stochastic differential equation with fractional Brownian motion input. Statist. Probab. Lett., 41(4):337-346, 1999.

[15] D. N. Keck and M. A. McKibben. On a McKean-Vlasov stochastic integro-differential evolution equation of Sobolev-type. Stochastic Anal. Appl., 21(5):1115-1139, 2003.

[16] H. Kunita. Stochastic flows and stochastic differential equations, volume 24 of Cambridge Studies in Advanced Mathematics. Cambridge University Press, Cambridge, 1990.

[17] S. J. Lin. Stochastic analysis of fractional Brownian motions. Stochastics Stochastics Rep., 55(1-2):121-140, 1995.

[18] Jiaowan Luo and Kai Liu. Stability of infinite dimensional stochastic evolution equations with memory and Markovian jumps. Stochastic Process. Appl., 118(5):864-895, 2008.

[19] Jiaowan Luo and Takeshi Taniguchi. The existence and uniqueness for non-Lipschitz stochastic neutral delay evolution equations driven by Poisson jumps. Stoch. Dyn., 9(1):135-152, 2009.

[20] N. I. Mahmudov and M. A. McKibben. Abstract second-order damped McKean-Vlasov stochastic evolution equations. Stoch. Anal. Appl., 24(2):303-328, 2006.

[21] Benoit B. Mandelbrot and John W. Van Ness. Fractional Brownian motions, fractional noises and applications. SIAM Rev., 10:422-437, 1968

[22] B. Maslowski and D. Nualart. Evolution equations driven by a fractional Brownian motion. J. Funct. Anal., 202(1):277-305, 2003.

[23] M. A. McKibben. Second-order damped functional stochastic evolution equations in Hilbert space. Dynam. Systems Appl., 12(3-4):467-487, 2003.

[24] M.A. McKibben and M. Webster. Abstract functional stochastic evolution equations driven by fractional brownian motion. Abstract and Applied Analysis, 2014(3):14 pages, 2014.

[25] M. Nagasawa and H. Tanaka. Diffusion with interactions and collisions between coloured particles and the propagation of chaos. Probab. Theory Related Fields, 74(2):161-198, 1987.

[26] B. Nagy. Cosine operator functions and the abstract Cauchy problem. Period. Math. Hungar., 7(3-4):213-217, 1976.

[27] Muthukumar Palanisamy and Rajivganthi Chinnathambi. Approximate controllability of second-order neutral stochastic differential equations with infinite delay and Poisson jumps. J. Syst. Sci. Complex., 28(5):1033-1048, 2015.

[28] A. Pazy. Semigroups of linear operators and applications to partial differential equations, volume 44 of Applied Mathematical Sciences. Springer-Verlag, New York, 1983. 
[29] Yong Ren and R. Sakthivel. Existence, uniqueness, and stability of mild solutions for second-order neutral stochastic evolution equations with infinite delay and Poisson jumps. J. Math. Phys., 53(7):073517, 14, 2012.

[30] C. C. Travis and G. F. Webb. Compactness, regularity, and uniform continuity properties of strongly continuous cosine families. Houston J. Math., 3(4):555-567, 1977.

[31] C. C. Travis and G. F. Webb. Cosine families and abstract nonlinear second order differential equations. Acta Math. Acad. Sci. Hungar., 32(1-2):75-96, 1978.

[32] V. V. Vasilev and S. I. Piskarev. Differential equations in Banach spaces. II. Theory of cosine operator functions. J. Math. Sci. (N. Y.), 122(2):3055-3174, 2004. Functional analysis. 